\title{
Corporate Citizenship
}

\section{Die Renaissance unternehmerischen Engagements}

\author{
Holger Backhaus-Maul, Christiane Biedermann, Stefan Nährlich und Judith Polterauer
}

Holger Backhaus-Maul ist Soziologe und Verwaltungswissenschaftler. Er ist wissenschaftlicher Mitarbeiter an der Martin-Luther-Universität HalleWittenberg und Vorstandsmitglied der Aktiven Bürgerschaft e. V., dem Kompetenzzentrum für Bürgerengagement der Volksbanken Raiffeisenbanken.

E-Mail holger.backhausmaul@paedagogik.uni-halle.de

Christiane Biedermann ist Sozialpädagogin und PR-Managerin. Sie verantwortet als Projektleiterin den Arbeitsbereich Corporate Citizenship, Presse und Kommunikation bei der Aktiven Bürgerschaft e. V. Dr. Stefan Nährlich ist Wirtschaftswissenschaftler und Geschäftsführer der Aktiven Bürgerschaft e. V. Judith Polterauer ist Soziologin, promoviert an der OttoFriedrich-Universität Bamberg zu Corporate Citizenship im Mittelstand und ist Projektleiterin des

Arbeitsbereichs Bürgergesellschaft der Aktiven Bürgerschaft e. V.

Internet

http://www.aktive-buergerschaft.de
Die internationale Debatte über Corporate Citizenship sieht Unternehmen nicht als rein wirtschaftliche Organisationen, sondern thematisiert deren neue gesellschaftliche Rolle und ihr freiwilliges Engagement. Als Corporate Citizen - als neue (Mit-) Spieler in der Zivilgesellschaft - finden Unternehmen auch in Deutschland seit einigen Jahren wachsende Aufmerksamkeit.

Über Unternehmen wurde und wird in Deutschland in der Öffentlichkeit häufig gesprochen. Seit einigen Jahren erfreuen sich »die Wirtschaft « und »die Unternehmen « geradezu einer gewissen Popularität, die mit einer eigenartigen Mischung aus öffentlicher Aufmerksamkeit, Ablehnung und Verantwortungszuweisung einhergeht.

Nach dem Zweiten Weltkrieg richtete sich in der kritischen Öffentlichkeit der alten Bundesrepublik das Augenmerk auf die politische Mitverantwortung von Wirtschaft und Unternehmen; zeitgleich wurden in der DDR Unternehmen aufgrund ideologischer Präferenzen verstaatlicht. In der alten Bundesrepublik wurde mit der Studentenbewegung der 1970er Jahre kapitalistisches Wirtschaften grundlegend diskreditiert und dann mit den Mitteln eines investigativen Journalismus »demaskiert «.

In Teilen der deutschen Öffentlichkeit so die klugen Beobachtungen des Berliner Sozialhistorikers Paul Nolte - hat sich eine grundlegend ablehnende Haltung gegenüber Wirtschaft und Unternehmen verfestigt. Begriffe wie »Neo-Liberalismus « und »Heuschrecken « markieren die vorläufigen Endpunkte dieser Diskussion. Passend dazu tragen einige namhafte Unternehmen in Deutschland durch ihr Handeln und Unterlassen - sei es in Siegerpose oder in Feudalherrenart - mit dazu bei, dass der Öffentlichkeit und den Medien die Anlässe zur Kritik nicht ausgehen.

Gleichzeitig zeigt sich seit Ende der 1990er Jahre in Deutschland - so etwa die politischen Analysen des Publizisten
Warnfried Dettling - ein wachsendes öffentliches Interesse an wirtschaftlichen Fragen und unternehmerischen Entwicklungen; die stille Popularität der Wirtschaftszeitschrift »brand eins « bringt diese Entwicklung treffend zum Ausdruck. Mit dem offensichtlichen Bedeutungsverlust staatlichen Handelns und Entscheidens einerseits und der bis ins Alltagsleben von Bürgern wirkenden Globalisierung des Wirtschaftens andererseits rücken Wirtschaft und Unternehmen insbesondere im Vergleich gegenüber Politik, Parteien und traditionellen Verbänden - in den Mittelpunkt des öffentlichen Interesses.

Damit wird die grundsätzliche Frage nach der Rolle von Unternehmen in der Gesellschaft virulent (vgl. BackhausMaul/Biedermann/Nährlich/Polterauer 2008). In Deutschland trifft die Suche von Unternehmen nach ihrer neuen Rolle in der Gesellschaft auf ein traditionsreiches, (sozial-) staatlich geprägtes Verständnis von Wirtschaft und Gesellschaft (»soziale Marktwirtschaft «). Demzufolge sind Unternehmen im Gesetzgebungsverfahren beteiligt, handeln mit Gewerkschaften Tarifverträge aus, engagieren sich im dualen Ausbildungssystem, führen in erheblichem Umfang Beiträge an das System der sozialen Sicherung ab und zahlen - mit "gewisser Varianz« - Steuern.

Darüber hinaus engagieren sich Unternehmen - was oft in Vergessenheit gerät in beachtlichem Maße freiwillig mittels Geld-, Sach- und Dienstleistungen in allen gesellschaftlich relevanten Bereichen (weiterführend Biedermann/Nährlich 2007). Erste empirische Studien weisen auf die Besonderheiten dieses freiwilligen gesellschaftlichen Engagements von Unternehmen in Deutschland hin. So sind es vor allem inhabergeführte Unternehmen, die sich in der Tradition eines deutschen Pfades von Corporate Citizenship verorten, wie etwa Bosch, Faber-Castell, Henkel oder Otto, um nur einige der be- 
kannten Namen exemplarisch zu nennen. Seit kurzem agieren auch kapitalmarktfinanzierte Unternehmen, allen voran »betapharm «, als profilierte Corporate Citizen. Gleichzeitig scheinen sich aber wohlbekannte und hoch gelobte Unternehmen aus den Anfängen der deutschen Corporate Citizenship-Debatte, wie etwa die Deutsche Telekom, Siemens oder Ford, getrieben von Korruptions- und Absatzproblemen schon wieder vom Spielfeld verabschiedet zu haben. Hierbei ist zu bedenken, dass die Nennung bekannter $\mathrm{Na}$ men im Bereich von Corporate Citizenship bisweilen eine tatsächlich wichtige Gruppe von Unternehmen in den Schatten stellt: die kleinst-, klein- und mittelständischen Unternehmen. Mittlerweile schließen sich diese Einzelakteure - wohlgemerkt ohne Beteiligung der konventionellen Unternehmens- und Arbeitgeberverbände - vor Ort und regional zu enga- gementbezogenen

Unternehmensnetzwerken zusammen.

Vor diesem Hintergrund einer traditionsreichen Unternehmenspraxis aus gesetzlich geregelten Pflichtleistungen und darüber hinausgehendem freiwilligen Engagement wurde in Deutschland die internationale Debatte über das gesellschaftliche Engagement von Unternehmen zunächst schlicht ignoriert oder als modische Erscheinung ohne substanziellen Neuigkeitswert (fehl-) interpretiert. Aber mittlerweile haben international gebräuchliche Begriffe wie Corporate Citizenship (gesellschaftliches Engagement von Unternehmen) und Corporate Social Responsibility (verantwortliche Unternehmensführung bei der Ausgestaltung betrieblicher Prozesse und Strukturen) Eingang in die deutsche Debatte gefunden (vgl. Backhaus-Maul 2008). Dieses dürfte nicht nur auf die bereits globalisierte

\section{Erfolgreiche Suchbewegungen}

»Als Fazit für VAUDE als Corporate Citizen ergibt sich, über die bereits beschriebenen positiven unternehmensinternen Effekte hinaus, Folgendes: VAUDE erweist sich als >Bürger mit inzwischen weithin anerkanntem sozialem Verantwortungsbewusstsein und sozialer Kompetenz. Über den Aufbau des Kinderhauses konnten viele Kontakte zu gesellschaftlichen Gruppen im Umfeld (z. B. Pfarrgemeinde, Stadt, Behörden etc.) erschlossen oder intensiviert werden. Die so geschaffene Wechselwirkung sorgt für eine starke und vertrauensvolle Verankerung des Unternehmens in der Gesellschaft, die in vielerlei Hinsicht für einen >Vertrauensbonus« sorgt. «

Antje von Dewitz, VAUDE

»Um eine möglichst breite Wirkung zu entfalten und weitere Partner aufzubauen, ist die Struktur des BildungsCent als gemeinnütziger Verein angelegt. Die Herlitz PBS AG als Gründungsmitglied übernimmt dabei die Rolle eines Inkubators. Neue Projektideen werden mit ihrer Unterstützung pilotiert und dann gemeinsam mit weiteren Unternehmenspartnern vergrößert. Bestes Beispiel ist das Programm 'Schule in Bewegung`, das inzwischen in enger $\mathrm{Zu}$ sammenarbeit mit der EDEKA in weit über 70 Schulen umgesetzt werden konnte. Gerade in der partnerschaftlichen Zusammenarbeit im Netzwerk liegt die Besonderheit der Arbeit des BildungsCent e. V. und wesentliches Ziel ist es, die Schulen bei der Umsetzung wichtiger Veränderungsprozesse zu unterstützen. Dazu bedarf es einer breiten gemeinsamen Basis, die es gleichzeitig erlaubt, die Kernkompetenzen und Interessen der unterstützenden Unternehmen einzubringen. Auf dieser Grundlage können die Unternehmen ihr Engagement aufbauen und gestalten, oder wenn sie, wie z. B. die KPMG Wirtschaftprüfungsgesellschaft oder die Deutsche Bank in ihren Corporate Volunteering Aktivitäten bereits sehr weit entwickelt sind, den BildungsCent e. V. als kompetente Mittlerorganisation nutzen.<

Silke Ramelow, Herlitz PBS AG/BildungsCent e. V.

Die Zitate stammen aus dem folgenden neuen Buch: Holger Backhaus-Maul, Christiane Biedermann, Judith Polterauer, Stefan Nährlich (Hg.): Corporate Citizenship in Deutschland. Verlag für Sozialwissenschaften, Wiesbaden 2008. 541 Seiten. 39,90 Euro. ISBN 978-3-531-15959-1.

Kommunikations- und Unternehmensberatungsbranche zurückzuführen sein, die einzelne, national begründete Managementinstrumente und -verfahren erfolgreich in unterschiedliche Länder exportiert. Sondern die mit wirtschaftlicher Globalisierung einhergehenden gesellschaftlichen Veränderungen stellen faktisch neue Anforderungen an Unternehmen in Deutschland.

\section{Globale Entwicklung}

Angesichts globaler wirtschaftlicher Prozesse und entsprechend tätiger Unternehmen erodieren die Handlungsspielräume von Nationalstaaten, die Teile ihrer staatlichen Entscheidungs- und Steuerungsfähigkeit verlieren oder abgeben. Vor diesem Hintergrund stehen auch die "Soziale Marktwirtschaft « und die traditionelle Unternehmensrolle des Sozialpartners als kostenträchtige, Löhne und Unternehmensgewinne belastende Faktoren zur Disposition.

Gleichwohl können sich Unternehmen aber nicht einfach auf ihr wirtschaftliches Kerngeschäft zurückziehen. Sie sind nach wie vor wirtschaftliche Organisationen in je spezifischen, sich dynamisch wandelnden Gesellschaften. Zweifelsohne ist die wirtschaftliche Tätigkeit Ausgangs- und Bezugspunkt unternehmerischen Handelns. Der wirtschaftliche Erfolg eines Unternehmens entscheidet über dessen Aufstieg und Fortbestand. Gegenüber diesem wirtschaftlichen Primärziel ist gesellschaftliches Engagement für Unternehmen nachrangig, gleichwohl aber nicht als »schmückendes Beiwerk «, "gelebtes Brauchtum « oder »Organisationsfolklore « fehl zu deuten.

Vielmehr stehen Unternehmen angesichts des sukzessiven Bedeutungswandels und Steuerungsverlustes von Nationalstaaten vor der Herausforderung, eigene Beiträge zur Human- und Sozialkapitalbildung sowie zur Gestaltung und Steuerung von Gesellschaft zu leisten. So kann das Wirtschaftssystem nicht mit der gleichen Selbstverständlichkeit wie früher einfach davon ausgehen, dass das staatliche Bildungs- und Erziehungssystem allein in ausreichender Menge und Qualität die gesellschaftliche $\mathrm{Hu}$ man- und Sozialkapitalbildung sicherstellt. Oder noch grundlegender formuliert: Engagement bietet Unterneh- 
men - jenseits der ausgetretenen Pfade politischer Einflussnahme - eine neuartige gesellschaftliche Möglichkeit und Verantwortung zur Mitentscheidung und Mitgestaltung. Dabei ist aber noch offen, welche staatlichen Steuerungs- und Leistungsdefizite - zumindest punktuell überhaupt kompensiert werden sollen und können.

\section{Internationale und deutsche Debatte}

Die internationale Debatte über Corporate Citizenship bricht mit der konventionellen Sichtweise, dass Unternehmen rein wirtschaftliche Organisationen sind. Zunehmend häufiger verweisen Unternehmen auf ihr Selbstverständnis und ihre Expertise als eigenständige und eigensinnige wirtschaftliche Organisationen, die bestrebt sind, sich in ihrer gesellschaftlichen Umwelt zu verorten. Genau diese selbst bestimmten Suchbewegungen von Unternehmen nach einer zeitgemäßen Rolle in der Gesellschaft bringt der Begriff »Corporate Citizenship« zum Ausdruck (vgl. die Beiträge in BackhausMaul/Biedermann/Nährlich/Polterauer 2008).

Bereits seit Ende der 1970er wird in den USA unter dem Begriff Corporate Citizenship über das gesellschaftliche Engagement von Unternehmen berichtet und diskutiert. Aber erst in den 1990er Jahren fand dieser Begriff auch Eingang in die entsprechenden Debatten in europäischen Ländern, allen voran Großbritannien, gefolgt von den Niederlanden und Dänemark. In Deutschland wurde er publizistisch erstmals 1995 von Achim Westebbe und David Logan verwendet, wobei es aber noch einige Jahre dauern sollte, bis er in der entsprechenden deutschen $\mathrm{Fa}$ chöffentlichkeit überhaupt Aufmerksamkeit fand. Anfangs dominierten in der deutschen (Medien-) Öffentlichkeit schlichte positive Darstellungen über »die guten Taten von Unternehmen«. Zugleich wurde - gesellschaftspolitisch äußerst folgenreich - der kritisch-liberale und zivilgesellschaftliche Gehalt der Corporate Citizenship-Debatte US-amerikanischer Prägung ausgeblendet, der u. a. in der Wahrnehmung von Freiheit und Verantwortung in der Community, der Pflege von Entrepreneurship- und Selbstorganisationskompetenzen sowie Respekt und Anerkennung gegenüber »Fremden « gründet.

Betrachtet man die Engagementpraxis von Unternehmen in Deutschland eingehender, so fällt zunächst auf, dass die klassischen Geld- und Sachspenden nach wie vor im Mittelpunkt stehen. Aber Engagementformen wie Stiftungen und insbesondere das von Unternehmen unterstützte Mitarbeiterengagement (Corporate Volunteering) gewinnen demgegenüber an Bedeutung. Die vielfältige und schillernde Realität des gesellschaftlichen Engagements von Unternehmen ist in Deutschland mangels entsprechender wissenschaftlicher Untersuchungen bisher aber nur unzureichend erfasst (vgl. Polterauer 2008).

Die aktuelle Diskussion über das gesellschaftliche Engagement von Unternehmen wird in Deutschland - trotz wohlklingender globaler Begriffe - bisweilen eher konventionell geführt: Ein geringes öffentliches Vertrauen in die Selbstregelungskompetenz von Unternehmen, latente gesellschaftliche Erwartungen an die Regulierungskompetenz des Staates und eine unternehmensferne Zivilgesellschaft markieren das Spielfeld. Und Unternehmen, die eigentlichen Spieler in diesem Engagementfeld, wirken als Ganzes bisweilen abwesend oder auch einfach nur träge. Insofern sind die Aktivitäten, Projekte und Programme derjenigen Unternehmen, die sich als Corporate Citizen engagieren, zunächst einmal anzuerkennen. Die Spiele der vergangenen Jahrzehnte waren aber auch für Unternehmen nur mäßig attraktiv, da sie vor allem von staatsbürgerlicher Pflichtenrhetorik, kultiviertem Neid, ritualisierten Kooperationsbehauptungen, kurzfristigen Gewinninteressen und wirtschaftlicher Naivität geprägt waren. Gleichwohl drängen sich mittlerweile am Ende der unternehmerischen Suchphase bei vielen Aktivitäten, Projekten und Programmen im Bereich Corporate Citizenship kritische wissenschaftliche und gesellschaftspolitische Fragen zur Ressourcenausstattung, Professionalität, Evaluation und vor allem zur gesellschaftlichen Bedeutung und Wirkungen auf.

\section{Resümee}

Soll Corporate Citizenship auch in Deutschland Erfolg haben und das gesell- schaftliche Engagement von Unternehmen ein dauerhafter Bestandteil in der gesellschaftlichen Aufgabenteilung sein, so sind kreative Suchbewegungen bei der Entwicklung dieses Feldes unerlässlich. Hier erkunden Unternehmen Handlungsoptionen, schätzen Erfolgsaussichten und Risiken ein und kalkulieren den notwendigen Ressourcenbedarf. Dabei verschließen sich unternehmerische Suchbewegungen direkten politischen Interventionen. Demgegenüber könnte eine wirtschaftlich interessierte und informierte sowie souveräne Zivilgesellschaft Unternehmen - in einem wohlverstandenen Eigeninteresse - gerade im Engagementbereich, in dem es im Kern um die Entwicklung von Human- und Sozialkapital geht, Chancen eröffnen um "gesellschaftliche Investitionsbrachen « zu erkennen und zu erschließen.

\section{Literatur}

Backhaus-Maul, Holger/Biedermann, Christiane/Polterauer, Judith/Nährlich, Stefan (Hg.) 2008: Corporate Citizenship in Deutschland, Wiesbaden: Verlag für Sozialwissenschaften.

Backhaus-Maul, Holger 2008: Traditionspfad mit Entwicklungspotenzial, in: Aus Politik und Zeitgeschichte, Heft 31, S. 14-20, Internet http://www.bpb. de/files/M5B1J7.pdf.

Biedermann, Christiane/Nährlich, Stefan 2007: Gemeinnützige Organisationen als Partner von Unternehmen. In: André Habisch/René Schmidpeter (Hg.): Handbuch Corporate Citizenship, Berlin/Heidelberg: Springer Verlag, S. 419-430.

Polterauer, Judith 2008: Corporate-Citizenship-Forschung in Deutschland, in: Aus Politik und Zeitgeschichte, Heft 31, S. 32-38, Internet http://www.bpb. de/files/M5B1J7.pdf. 